\title{
Effects of rare earth ion size on the stability of the coherent Jahn-Teller distortions in undoped perovskite manganites
}

\author{
T. F. Seman, ${ }^{1}$ K. H. Ahn,${ }^{1}$ T. Lookman,${ }^{2}$ A. Saxena,${ }^{2}$ A. R. Bishop, ${ }^{2}$ and P. B. Littlewood ${ }^{3}$ \\ ${ }^{1}$ Department of Physics \\ New Jersey Institute of Technology \\ Newark, New Jersey 07102, USA \\ ${ }^{2}$ Theoretical Division \\ Los Alamos National Laboratory \\ Los Alamos, New Mexico 87545, USA \\ ${ }^{3}$ Physical Sciences and Engineering Division \\ Argonne National Laboratory \\ Argonne, Illinois 60439, USA
}

\begin{abstract}
We present a theoretical study on the relation between the size of the rare earth ions, often known as chemical pressure, and the stability of the coherent Jahn-Teller distortions in undoped perovskite manganites. Using a Keating model expressed in terms of atomic scale symmetry modes, we show that there exists a coupling between the uniform shear distortion and the staggered buckling distortion within the Jahn-Teller energy term. It is found that this coupling provides a mechanism by which the coherent Jahn-Teller distortion is more stabilized by smaller rare earth ions. We analyze the appearance of the uniform shear distortion below the Jahn-Teller ordering temperature, estimate the Jahn-Teller ordering temperature and its variation between $\mathrm{NdMnO}_{3}$ and $\mathrm{LaMnO}_{3}$, and obtain the relations between distortions. We find good agreement between theoretical results and experimental data.
\end{abstract}

PACS numbers: 75.47.Gk, 74.62.Dh, 64.70.K-, 61.50.Ks 


\section{INTRODUCTION}

Since the discovery of the colossal magnetoresistance effect, a lot of attention has focused on a class of materials known as perovskite manganites. ${ }^{1-6}$ These materials have the chemical formula in the form of $R E_{1-x} A K_{x} \mathrm{MnO}_{3}$, where $R E$ and $A K$ represent the rare earth and alkali metal elements, and have a perovskite structure. One of the major research themes for these materials is the relation between their physical properties and the average size of ions at the $R E / A K$ site, often known as the chemical pressure effect. The size of the $R E / A K$ ion is usually parameterized by a tolerance factor and one of the most important phase diagrams for these materials has been the one in the temperature versus tolerance factor plane for a fixed $30 \%(x=0.3)$ doping ratio ${ }^{\underline{7}}$ The $R E / A K$ ions with size smaller than the space created by the surrounding $\mathrm{MnO}_{6}$ octahedra induce buckling of the Mn-O-Mn bonds, observed through various structural refinement analyses.

How this buckling distortion affects the properties of manganites has been controversial. It is well known from experimental observations that there is strong competition between the insulating phase with a coherent Jahn-Teller (JT) distortion and the metallic phase without such distortion. $\underline{\underline{6}}$ So far, most attention has centered on the impact of the buckling on the metallic phase, in particular, the possible change in the effective Mn-O-Mn electron hopping parameter and the band width.$^{7}$ However, there has been a debate on whether the change of the hopping parameter due to the Mn-O-Mn bond angle change of several

degrees would be significant enough to explain the observed metal-insulator transition. $\underline{-1}^{-12}$ A less studied effect of the Mn-O-Mn bond buckling, except for a few early efforts based on

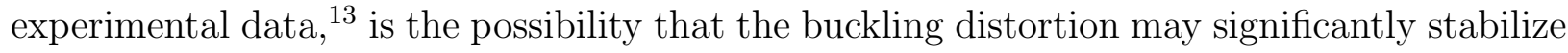
the insulating phase with a coherent JT distortion. The main goal of this paper is to examine such a possibility with a simplified model of the perovskite manganites. To be specific, we analyze the interplay between the JT ordering and chemical pressure for undoped perovskite manganites. The study on undoped manganites is merited because they are not only parent compounds of doped perovskite manganites, but also because one of the first multiferroic materials discovered is an undoped manganite, $\mathrm{TbMnO}_{3}$, with a relatively small rare earth element. 14 Therefore, the chemical pressure effect in undoped manganites reported in this paper would also be relevant for future studies on how the multiferroic property appears in $R E \mathrm{MnO}_{3}$ with small $R E$ ions, as well as for the effect of chemical pressure on the distorted 


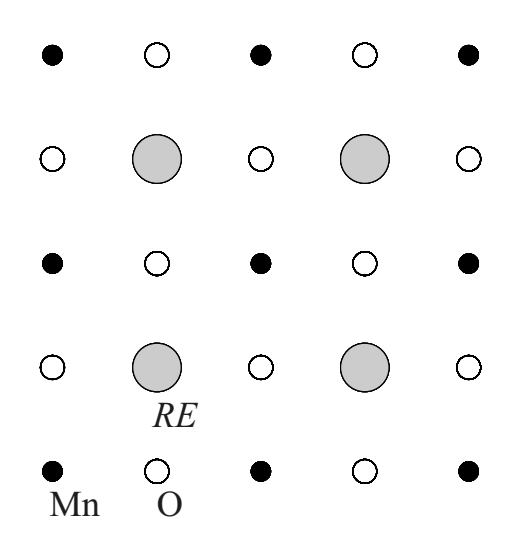

FIG. 1. Two-dimensional model for the perovskite structure considered in the text.

insulating phase of doped manganites.

\section{MODEL SYSTEM AND ENERGY EXPRESSION}

We study a two-dimensional (2D) model for the perovskite structure which incorporates both buckling and the JT distortions. We define a 2D perovskite structure shown in Fig. 1 which includes the following aspects of the 3D perovskite structure for undoped manganites: (1) symmetry breaking distortion of oxygen ions around Mn ion, (2) chemical pressure effect, which is the attraction of surrounding oxygen ions toward the small rare earth ions, and (3) the rotation of oxygen ions with alternating directions around Mn ions, which is, in effect, the buckling of Mn-O-Mn bonds.

We apply the recently developed atomic scale description of lattice distortions $\frac{15}{1}, \underline{16}$ to describe the elastic energy of the system. In this approach, atomic scale modes of lattice distortions and their constraints are used instead of displacement variables. The structural motifs can be chosen in any convenient way as long as they have the symmetry of the crystal structure. We choose two "structural motifs", shown in Fig. 2: one consists of one $\mathrm{Mn}$ ion and four surrounding $\mathrm{O}$ ions and the other comprises of one rare earth $(R E)$ ion and four surrounding $\mathrm{O}$ ions. We obtain seven symmetry modes for each motif, shown in Fig. 3 for the $\mathrm{MnO}_{4}$ motif. $\frac{17}{\underline{17}}$ Similar symmetry modes are defined for the $R E \mathrm{O}_{4}$ motif and are distinguished with primes on the symbols in this paper. The modes defined for each plaquette on the lattice are constrained by each other because neighboring motifs share

ions, which leads to constraint equations between the Fourier components of the modes. In terms of these fourteen modes and constraint equations, any distortion of the 2D perovskite 


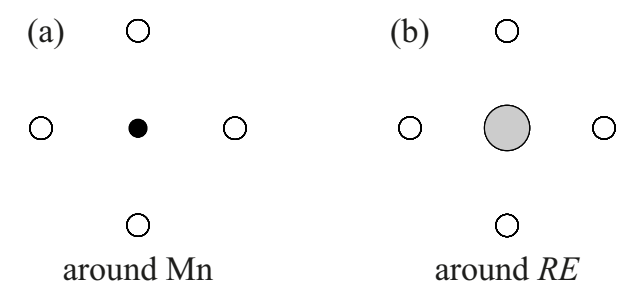

FIG. 2. Two structural motifs chosen for the 2D structure shown in Fig. 1,

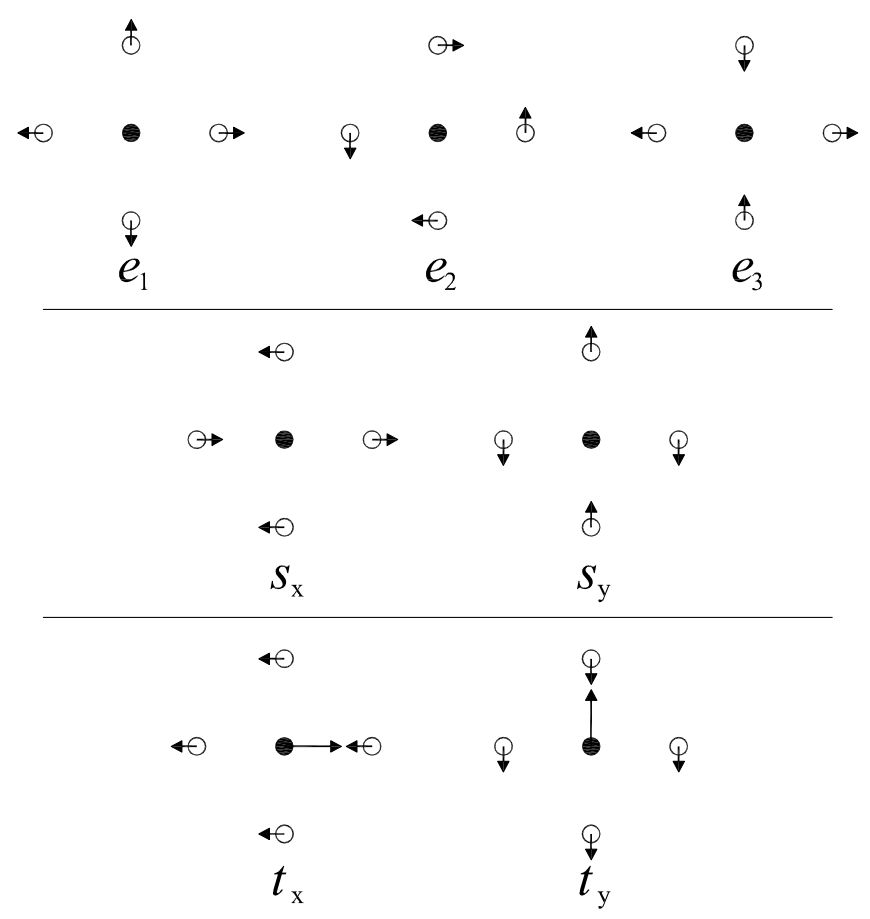

FIG. 3. Distortion modes for the motif around the Mn ion in Fig. 2, Similar distortion modes, $e_{1}^{\prime}$, $e_{2}^{\prime}, e_{3}^{\prime}, s_{x}^{\prime}, s_{y}^{\prime}, t_{x}^{\prime}, t_{y}^{\prime}$ are defined for the motif around the $R E$ ion.

structure shown in Fig. 1 can be described.

For the current study, since we are interested in the ordered state, we consider distortions with wavevectors $\vec{k}=(0,0)$ and $(\pi, \pi)$ only. For these wavevectors, the constraint equations are as follows, where we use subscripts 0 and $s$ to represent $\vec{k}=(0,0)$ and $(\pi, \pi)$, respectively: $e_{10}=e_{10}^{\prime}, e_{20}=e_{20}^{\prime}, e_{30}=e_{30}^{\prime}, s_{x 0}=-s_{x 0}^{\prime}, s_{y 0}=-s_{y 0}^{\prime}, e_{1 s}=-e_{2 s}^{\prime}, e_{1 s}^{\prime}=-e_{2 s}, s_{x s}=s_{y s}=$ $s_{x s}^{\prime}=s_{y s}^{\prime}=0$. Rest of the modes are unconstrained, particularly, $e_{3 s}$ and $e_{3 s}^{\prime}$. We search for the interplay between the staggered deviatoric distortion mode $e_{3 s}$ and the staggered rotation of $\mathrm{O}$ ions around $\mathrm{Mn}$ ion (or equivalently staggered Mn-O-Mn bond buckling mode) $e_{3 s}^{\prime}$, where the latter is due to the compression $e_{10}^{\prime}=e_{10}$ by small rare earth ions. Therefore, 


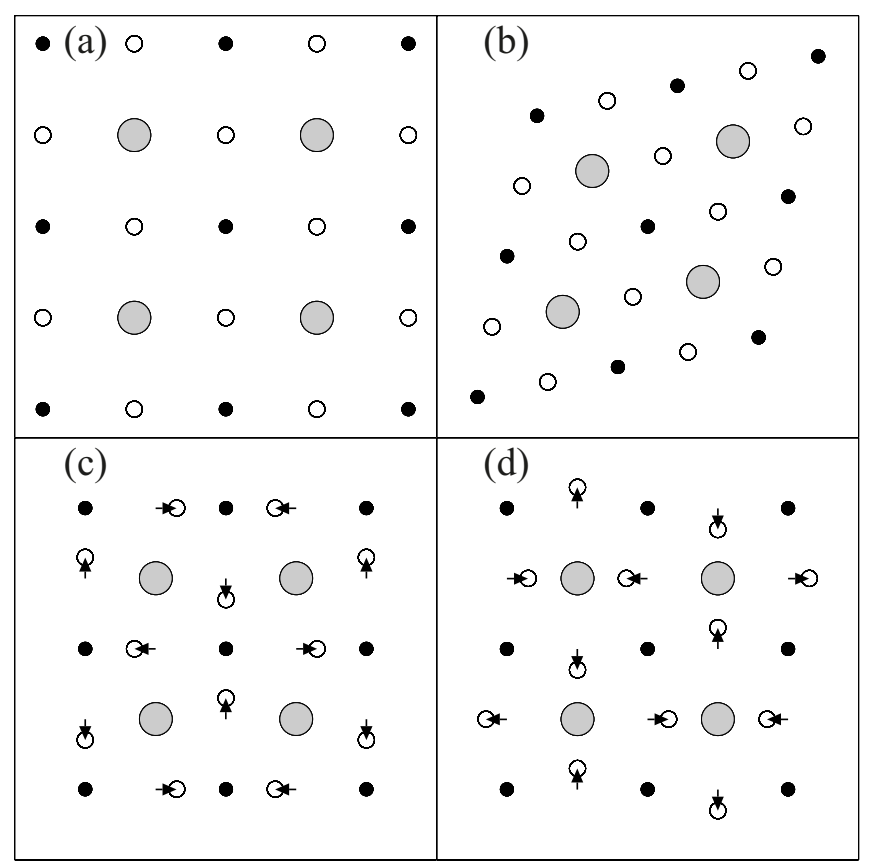

FIG. 4. Four distortion modes considered in the current study: (a) uniform dilatation mode $e_{10}$, (b) uniform shear mode $e_{20}$, (c) staggered deviatoric mode $e_{3 s}$, (d) staggered buckling mode $e_{3 s}^{\prime}$. All figures are drawn for the positive values of the modes with the Mn site at the left bottom corner chosen as the origin.

we limit ourselves to the modes $e_{10}^{\prime}=e_{10}, e_{20}=e_{20}^{\prime}, e_{3 s}$, and $e_{3 s}^{\prime}$, shown in Fig. 4. We include the uniform shear mode $e_{20}=e_{20}^{\prime}$ because it is coupled to $e_{3 s}^{\prime}$ through the JT term, as will be discussed later in this paper.

Even though it is possible to analyze an energy expression including higher order symmetry-allowed anharmonic energy terms, such a method would generate many parameters and would make the model less predictive. Therefore, we start with a Keating model with a small number of parameters, $\stackrel{18,19}{19}$ and map the Keating model onto the approach based on the symmetry modes. In the Keating approach, the elastic energy is represented in terms of bond length and bond angle changes from equilibrium. For our 2D perovskite structure, we consider the following set of Keating variables and harmonic moduli for each Mn ion, as shown in Fig. 5: $\delta l_{n}(n=1,2,3,4)$ and modulus $a_{1}$ for Mn-O bond length change, $\delta \theta_{n}(n=1,2,3,4)$ and $b_{1} / 4$ for $90^{\circ}$ O-Mn-O bond angle change, $\delta r_{n}(n=1,2,3$, 4) and $a_{2}$ for $R E$-O bond length change, and $\delta \varphi_{n}(n=1,2)$ and $b_{2} / 4$ for $180^{\circ} \mathrm{Mn}-\mathrm{O}-\mathrm{Mn}$ bond angle change. We note that the $\mathrm{MnO}_{4}$ motif is considered as relatively stiff compared 


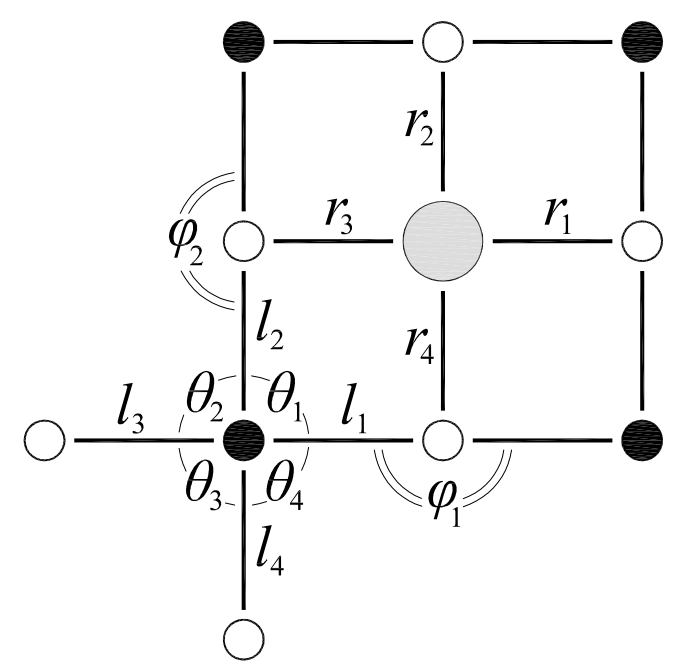

FIG. 5. The Keating variables considered for each Mn ion. $l_{1}, l_{2}, l_{3}$, and $l_{4}$ represent the Mn-O bond lengths. $\theta_{1}, \theta_{2}, \theta_{3}$, and $\theta_{4}$ represent the O-Mn-O bond angles. $r_{1}, r_{2}, r_{3}$, and $r_{4}$ represent $R E-\mathrm{O}$ bond lengths. $\varphi_{1}$ and $\varphi_{2}$ indicate Mn-O-Mn bond angles.

to other components of the structure, so that $a_{1} \gg a_{2}$ and $b_{1} \gg b_{2}$.

We consider the following Keating elastic energy expression per Mn ion,

$$
\begin{aligned}
E_{\text {elastic }} & =\frac{1}{2} a_{1} \sum_{n=1,2,3,4}\left(\delta l_{n}\right)^{2}+\frac{1}{2} b_{1} \sum_{n=1,2,3,4}\left(\delta \theta_{n} / 2\right)^{2} \\
& +\frac{1}{2} a_{2} \sum_{n=1,2,3,4}\left(\delta r_{n}\right)^{2}+\frac{1}{2} b_{2} \sum_{n=1,2}\left(\delta \varphi_{n} / 2\right)^{2} .
\end{aligned}
$$

We express the Keating variables in terms of $e_{10}, e_{20}, e_{3 s}$ and $e_{3 s}^{\prime}$. For example, we obtain

$$
\begin{gathered}
\delta l_{1}=\frac{\sqrt{\left(1+e_{10}+e_{3 s}\right)^{2}+\left(e_{20}+e_{3 s}^{\prime}\right)^{2}}-1}{2} \\
\delta \theta_{1}=\tan ^{-1}\left(\frac{e_{20}+e_{3 s}^{\prime}}{1+e_{10}+e_{3 s}}\right)+\tan ^{-1}\left(\frac{e_{20}-e_{3 s}^{\prime}}{1+e_{10}-e_{3 s}}\right) .
\end{gathered}
$$

The Taylor expansion of $E_{\text {elastic }}$ in terms of $e_{10}, e_{20}, e_{3 s}$ and $e_{3 s}^{\prime}$ produces all the terms of any order. We make an approximation that $b_{2}$ is much smaller than other parameters, as mentioned above, and drop the terms with $b_{2}$. We keep all harmonic order terms and select the cubic and quartic order terms that are responsible for the Mn-O-Mn bond buckling instability, which are shown below as $E_{\text {har }}, E_{\text {cubic }}$, and $E_{\text {quartic }}$.

We further define the JT energy per $\mathrm{Mn}$ ion $E_{\mathrm{JT}}$ and the energy associated with the tolerance factor per $\mathrm{Mn}$ ion $E_{\text {tol }}$ as follows:

$$
E_{\mathrm{JT}}=-\frac{\lambda}{2}\left|\delta l_{1}+\delta l_{3}-\delta l_{2}-\delta l_{4}\right|
$$




$$
E_{\mathrm{tol}}=\frac{\tilde{p}}{2}\left(\delta r_{1}+\delta r_{2}+\delta r_{3}+\delta r_{4}\right)
$$

where we define "chemical pressure"

$$
\tilde{p}=C_{1}^{\prime}(1-t)
$$

The parameter $t$ is a two-dimensional analog of the tolerance factor for the 3D perovskite structure, and the coefficient $C_{1}^{\prime}$ represents the coupling between the average $R E$-O bond length and the tolerance factor $t$. The "chemical pressure" $\tilde{p}$ induces the shortening of the average $R E-\mathrm{O}$ bond length due to small $R E$ ions. We also define the JT distortion mode

$$
e_{\mathrm{JT}}=\left(\delta l_{1}+\delta l_{3}-\delta l_{2}-\delta l_{4}\right) / 2
$$

which represents the anisotropic bond length change, similar to the 3D JT distortion modes, often written as $Q_{2}$ and $Q_{3}$ (Ref. 20). The expression $E_{\mathrm{JT}}=-\lambda\left|e_{\mathrm{JT}}\right|$ is based on the $3 \mathrm{D}$ JT energy $E_{\mathrm{JT}, 3 \mathrm{D}}=-\lambda_{Q} \sqrt{Q_{2}^{2}+Q_{3}^{2}}$ which is obtained after minimizing $\mathrm{JT}$ electronlattice coupling energy in manganites with respect to the $e_{g}$ orbital state ${ }^{20}$ In undoped manganites, $Q_{3} / Q_{2}$ is about 0.3 - 0.4 (Refs. 21 and 22), which allows an approximation $E_{\mathrm{JT}, 3 \mathrm{D}} \approx-\lambda_{Q}\left|Q_{2}\right|\left[1+\left(Q_{3} / Q_{2}\right)^{2} / 2\right]$. Further neglecting the small $\left(Q_{3} / Q_{2}\right)^{2} / 2$ term, we get the $2 \mathrm{D}$ analog of the JT energy $E_{\mathrm{JT}}$, with the $2 \mathrm{D}$ JT distortion $e_{\mathrm{JT}}$ corresponding to $3 \mathrm{D} \mathrm{JT}$ distortion $Q_{2}$ except for a normalization factor difference.

We expand $E_{\mathrm{JT}}$ and $E_{\mathrm{tol}}$ in the form of a Taylor series in $e_{10}, e_{20}, e_{3 s}$ and $e_{3 s}^{\prime}$. Only the leading order energy terms being kept, our total energy expression per $\mathrm{Mn}$ ion $E_{\text {tot }}$ is given below.

$$
\begin{aligned}
E_{\mathrm{tot}}= & E_{\mathrm{har}}+E_{\mathrm{JT}}+E_{\mathrm{tol}}+E_{\mathrm{cubic}}+E_{\mathrm{quartic}}, \\
E_{\mathrm{har}}= & \frac{1}{2}\left(a_{1}+a_{2}\right)\left(e_{10}\right)^{2}+\frac{1}{2}\left(4 b_{1}\right)\left(e_{20}\right)^{2} \\
& +\frac{1}{2} a_{1} e_{3 s}^{2}+\frac{1}{2} a_{2}\left(e_{3 s}^{\prime}\right)^{2} \\
E_{\mathrm{JT}}= & -\lambda\left|e_{3 s}+e_{20} e_{3 s}^{\prime}\right| \\
E_{\mathrm{tol}}= & \tilde{p} e_{10} \\
E_{\text {cubic }}= & \frac{1}{2} a_{1} e_{10}\left(e_{3 s}^{\prime}\right)^{2} \\
E_{\text {quartic }}= & \frac{1}{4} \frac{a_{1}}{2}\left(e_{3 s}^{\prime}\right)^{4}
\end{aligned}
$$

where the relation

$$
e_{\mathrm{JT}} \approx e_{3 s}+e_{20} e_{3 s}^{\prime}
$$


is used for $E_{\mathrm{JT}}$. The physical origin of the coupling between $e_{20}$ and $e_{3 s}^{\prime}$ is important for the current study and is explained in more detail in Sect. VA.

\section{ESTIMATION OF PARAMETERS}

In this section, we present our estimate of the parameters. We choose the Mn-Mn distance before the distortion, which is around $u=4 \AA$, as 1 . Therefore, $e_{10}, e_{20}, e_{3 s}$, and $e_{3 s}^{\prime}$ are unitless, and $a_{1}, b_{1}, a_{2}, b_{2}$, and $\lambda$ have the unit of energy. The parameter $a_{1}$ can be estimated from the Mn-O bond stretching phonon mode energy, which is about $70 \mathrm{meV}$ from optical measurements. ${ }^{20}$ From $\hbar \sqrt{2 a_{1} / m_{\mathrm{O}}}=70 \mathrm{meV}$ with $m_{\mathrm{O}}$ the mass of the oxygen ion, we obtain $a_{1} \approx 150 \mathrm{eV}$. We estimate $b_{1}$ from the elastic modulus, $c_{44}$. From Ref. $23, c_{44} \approx 55-60 \mathrm{GPa}$. The uniform shear mode $e_{20}$ corresponds to the conventional $e_{x y} / 2$ (Ref. 24). Using the identity $1 \mathrm{GPa} \AA^{3}=6.3 \mathrm{meV}$, we find $b_{1} \approx 20-25 \mathrm{eV}$. To estimate $b_{2}$, we use the results ${ }^{25}$ for $\mathrm{ReO}_{3}$, which has no $R E / A K$ ion and, therefore, $a_{2}=0$ and the buckling of Re-O-Re bond depends only on $b_{2}$. According to the analysis in Ref. 25, the oxygen oscillation along Re-ORe direction has the angular frequency $\omega_{o}^{x}=905 \mathrm{~cm}^{-1}$, whereas the oscillation perpendicular to Re-O-Re direction has the angular frequency $\omega_{o}^{y}=30 \mathrm{~cm}^{-1}$, from which we can estimate $b_{2} / a_{1}=\left(\omega_{o}^{y} / \omega_{o}^{x}\right)^{2} / 2 \approx 0.5 \times 10^{-3}$. We can expect a similar order of magnitude for $b_{2}$ in manganites, order of $10^{-3} a_{1}$, for example, $0.2 \mathrm{eV}$, which is negligible compared to other parameter values and justifies neglecting the terms with $b_{2}$ as mentioned above. Various probes, such as neutron or optical spectroscopy, indicate the buckling mode frequency in manganites of about 35 - $50 \mathrm{meV}$ (Ref. 26). From the analysis of $(\pi, \pi)$ phonon mode for our model, we obtain the frequency of buckling mode $\omega_{\mathrm{bk}}=\sqrt{\left(2 a_{2}+4 b_{2}\right) / m_{\mathrm{O}}}$. Therefore, we obtain $a_{2} \approx 30-80 \mathrm{eV}$. For the estimation of $\lambda$, we match the JT energy gain for our 2D model with that for the 3D model to ensure that our 2D model represents the energy scale of the 3D materials correctly. For our 2D model $\Delta E_{\mathrm{JT}}=-\lambda^{2} /\left(2 a_{1}\right)$. For the 3D model in Ref. $20, \Delta E_{\mathrm{JT}} \approx-0.29 \mathrm{eV}$, and therefore, we obtain $\lambda \approx 10 \mathrm{eV}$. 


\section{INTERPLAY BETWEEN Mn-O-Mn BOND BUCKLING AND THE JAHN- TELLER DISTORTIONS}

\section{A. Buckling instability without the Jahn-Teller term}

We find the condition for the buckling instability without the effect of the JT energy term $E_{\mathrm{JT}}$. We take a perturbative approach rather than try to solve high order polynomial equations. By minimizing $E_{\mathrm{har}}+E_{\mathrm{tol}}$, we obtain

$$
\left(e_{10}\right)^{\min , *}=-\frac{\tilde{p}}{a_{1}+a_{2}},
$$

where the superscript * indicates that the JT term is not yet taken into consideration. This isotropic compression of the $\mathrm{MnO}_{4}$ motif renormalizes the coefficient of the $\left(e_{3 s}^{\prime}\right)^{2}$

term through the $E_{\text {cubic }}$ term. From this, we obtain the critical condition for the buckling instability,

$$
\begin{aligned}
\tilde{p}_{c}^{*} & =\frac{a_{2}}{a_{1}}\left(a_{1}+a_{2}\right), \\
\left(e_{10}\right)_{c}^{\min , *} & =-\frac{a_{2}}{a_{1}} .
\end{aligned}
$$

If $\tilde{p}>\tilde{p}_{c}^{*}$, Mn-O-Mn bond buckling occurs and the quartic order term, $E_{\text {quartic }}$, should be considered for the equilibrium $e_{3 s}^{\prime}$,

$$
\begin{aligned}
\left|\left(e_{3 s}^{\prime}\right)^{\min , *}\right| & =\sqrt{\frac{2}{a_{1}+a_{2}}} \sqrt{\tilde{p}-\tilde{p}_{c}^{*}} \\
& =\sqrt{2} \sqrt{\left(e_{10}\right)_{c}^{\min , *}-\left(e_{10}\right)^{\min , *}} .
\end{aligned}
$$

The minimized $E_{\text {tot }}$ without the $E_{\mathrm{JT}}$ term is given by

$$
E_{\mathrm{tot}}^{\min , *}=-\frac{\tilde{p}^{2}}{2\left(a_{1}+a_{2}\right)}-\frac{a_{1}}{2}\left(\frac{\tilde{p}}{a_{1}+a_{2}}-\frac{a_{2}}{a_{1}}\right)^{2} .
$$

\section{B. Buckling instability with the Jahn-Teller term}

We now examine how the JT energy term $E_{\mathrm{JT}}$ alters the buckling instability. From $E_{\text {har }}+E_{\text {tol }}+E_{\mathrm{JT}}$, we obtain

$$
\begin{aligned}
& \left(e_{10}\right)^{\min }=-\frac{\tilde{p}}{a_{1}+a_{2}}, \\
& \left(e_{3 s}\right)^{\min }=\frac{\lambda}{a_{1}},
\end{aligned}
$$


where we consider the $\left(e_{3 s}\right)^{\text {min }}>0$ case only. The buckling instability is found from the second order terms in $e_{20}$ and $e_{3 s}^{\prime}$ in $E_{\text {tot }}$ :

$$
\frac{1}{2}\left(4 b_{1}\right)\left(e_{20}\right)^{2}+\frac{1}{2}\left[a_{2}+a_{1}\left(e_{10}\right)^{\min }\right]\left(e_{3 s}^{\prime}\right)^{2}-\lambda e_{20} e_{3 s}^{\prime},
$$

where we assumed $\left(e_{3 s}\right)^{\text {min }}+e_{20} e_{3 s}^{\prime}>0$. From the condition $4 b_{1}\left[a_{2}+a_{1}\left(e_{10}\right)^{\text {min }}\right]<\lambda^{2}$, we obtain the critical condition

$$
\tilde{p}_{c}=\frac{a_{2}}{a_{1}}\left(a_{1}+a_{2}\right)-\frac{\lambda^{2}}{4 b_{1} a_{1}}\left(a_{1}+a_{2}\right)
$$

and the buckling distortion occurs for $\tilde{p}>\tilde{p}_{c}$. Comparing with $\tilde{p}_{c}^{*}$ in Eq. (16), we find that the JT energy makes buckling more likely. After this buckling instability, we should include the $E_{\text {quartic }}$ term to find the equilibrium result. For this, we first minimize $E_{\text {tot }}$ with respect to the shear distortion $e_{20}$ to obtain

$$
\left(e_{20}\right)^{\min }=\frac{\lambda}{4 b_{1}} e_{3 s}^{\prime} .
$$

Inserting this back, we get an energy expression for $E_{\text {tot }}$ only in terms of $e_{3 s}^{\prime}$, which gives the equilibrium buckling distortion and the minimum energy,

$$
\begin{gathered}
\left(e_{3 s}^{\prime}\right)^{\min }=\sqrt{\frac{2}{a_{1}+a_{2}}} \sqrt{\tilde{p}-\tilde{p}_{c}}, \\
E_{\mathrm{tot}}^{\min }=-\frac{\tilde{p}^{2}}{2\left(a_{1}+a_{2}\right)}-\frac{\lambda^{2}}{2 a_{1}} \\
-\frac{a_{1}}{2}\left(\frac{\tilde{p}}{a_{1}+a_{2}}-\frac{a_{2}}{a_{1}}+\frac{\lambda^{2}}{4 b_{1} a_{1}}\right)^{2} .
\end{gathered}
$$

Therefore, the energy gain due to the JT energy term is given by

$$
\Delta E_{\mathrm{JT}}=-\frac{\lambda^{2}}{2 a_{1}}-\frac{\left(\tilde{p}-\tilde{p}_{c}^{*}\right) \lambda^{2}}{4\left(a_{1}+a_{2}\right) b_{1}}
$$

up to order $\lambda^{2}$. The second term corresponds to the part of $\Delta E_{\mathrm{JT}}$ which depends on the size of rare earth ion, or $\tilde{p}$. This result shows that the small rare earth ion, or large chemical pressure, stabilizes the JT distortion.

\section{COMPARISON WITH EXPERIMENTS}

We make comparisons between our model and experimental results. In Sect. VA, we explain the simultaneous appearance of the uniform shear distortion and the long range JT 
distortion observed in undoped manganites $\underline{\underline{21}}$ In Sect. $\mathrm{VB}$, we estimate the changes in the JT ordering temperature $T_{\text {JT }}$ between $\mathrm{LaMnO}_{3}$ and $\mathrm{NdMnO}_{3}$, and compare with experiments. In Sect. VC, we calculate the ratios between different distortion modes and compare with experimental data for $\mathrm{LaMnO}_{3}$ and $\mathrm{NdMnO}_{3}$.

\section{A. Appearance of uniform shear distortion below the Jahn-Teller ordering tem-} perature

Experimental data in Refs. 21 and 22 show that the difference between the lattice constant $a$ and $b$ along the diagonal directions in the plane appears simultaneously with the long range JT distortion below $T_{\text {JT }}$ for both $\mathrm{LaMnO}_{3}$ and $\mathrm{NdMnO}_{3}$. This distortion corresponds to the uniform shear distortion in our model, related by $e_{20}=(b-a) /(2 \sqrt{2} u)$ with $u=4 \AA$. We analyze the coupling between the JT distortion and the uniform shear distortion, which is important for the stabilization of JT ordered state by the chemical pressure. In our model, such coupling originates from the term $e_{20} e_{3 s}^{\prime}$ in $e_{\mathrm{JT}}$ in Eq. (14) or in $E_{\mathrm{JT}}$ in Eq. (10), which can be understood as follows. We consider applying a positive $e_{20}$ shear distortion to the lattice, as shown in Fig. [ by the axis of elongation and compression along $45^{\circ}$ and $135^{\circ}$, respectively. Such uniform shear distortion makes the Mn-O bond lengths either longer or shorter depending on whether the direction of the bond is closer to the orientation of elongation $\left(45^{\circ}\right)$ or compression $\left(135^{\circ}\right)$, except for the bonds with directions right between the two directions. If the system does not have $(\pi, \pi)$ buckling, as shown in the thin solid lines in Fig. 6, all Mn-O bonds make equal angles from the axis of elogation/compression, and therefore $e_{20}$ shear distortion keeps all Mn-O bond lengths equal. This implies that $e_{20}$ distortion alone does not contribute to the JT distortion or JT energy gain. In contrast, if the system has a buckling distortion $e_{3 s}^{\prime}$ with a wave vector $\vec{k}=(\pi, \pi)$, as shown in the thick solid lines in Fig. 6, the $e_{20}$ shear distortion elongates Mn-O bonds marked with $l$ and shortens Mn-O bonds marked with $s$, depending on whether the bond direction is closer to the axis of elongation or the axis of compression, which results in the JT distortion $e_{\mathrm{JT}}$ with a wave vector $\vec{k}=(\pi, \pi)$. If this extra JT distortion is in the same [opposite] phase as [to] the deviatoric $e_{3 s}$ distortion, in other words, if $e_{20} e_{3 s}^{\prime}$ and $e_{3 s}$ have the same [opposite] sign, this extra JT distortion increases [decreases] the net JT distortion, which explains the expression of $E_{\mathrm{JT}}$ in Eq. (10) or $e_{\mathrm{JT}}$ in Eq. (14). We emphasize here that the extra 


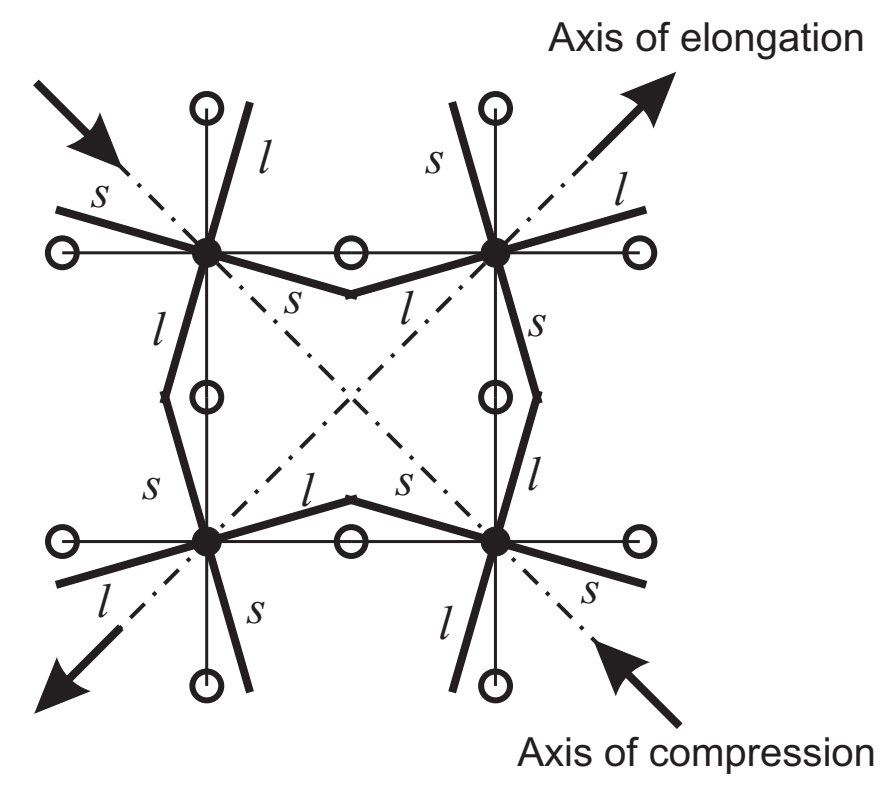

FIG. 6. Superposition of $(\pi, \pi)$ buckling $e_{3 s}^{\prime}$ and uniform shear distortion $e_{20}$ effectively generates the extra $(\pi, \pi)$ JT distortion of Mn-O bond lengths, as indicated by the $s$ and $l$ for the shortened and elongated bonds, which is responsible for the $e_{20} e_{3 s}^{\prime}$ coupling within $e_{\mathrm{JT}}$ and the Jahn-Teller coupling $E_{\mathrm{JT}}$. In the $(\pi, \pi) \mathrm{JT}$ ordered state, this adds up to the $e_{3 s}$ deviatoric mode if $e_{3 s}$ and $e_{20} e_{3 s}^{\prime}$ have the same sign. This mechanism is responsible for the appearance of the uniform shear distortion below the JT ordering temperature, as explained in Sect VA. The extra JT energy gain for the buckled lattice is responsible for the increase in $T_{\mathrm{JT}}$ in $R E \mathrm{MnO}_{3}$ with small $R E$ ions, as explained in Sect $\mathrm{VB}$

JT energy gain occurs only when the $e_{2}, e_{3}$ and $e_{3}^{\prime}$ distortions are in the right phase with respect to each other. Experiments ${ }^{21}$ show that the $(\pi, \pi)$ Mn-O-Mn bond buckling persists even above $T_{\mathrm{JT}}$ without much change in size. However, above $T_{\mathrm{JT}}$, the coherent $e_{3}$ distortion does not exist, and therefore the extra JT distortion due to the uniform $e_{20}$ distortion in the presence of staggered buckling distortion would increase the JT energy gain in some regions and decrease the JT energy gain in other regions, and does not change the net JT energy. In other words, the energy gain due to the cooperative effect between $e_{3}, e_{3}^{\prime}$, and $e_{2}$ does not exist at $T>T_{\mathrm{JT}}$. We therefore expect that the $e_{20}$ mode does not exist above $T_{\mathrm{JT}}$ and appears simultaneously with the long range JT ordering, consistent with the experimental results. 


\section{B. Jahn-Teller ordering temperature and its variation between $\mathrm{LaMnO}_{3}$ and} $\mathrm{NdMnO}_{3}$

It is reported 27 that $T_{\mathrm{JT}}$ changes from $750 \mathrm{~K}$ for $\mathrm{LaMnO}_{3}$ to $1100 \mathrm{~K}$ for $\mathrm{NdMnO}_{3}$ by about $\Delta T_{\mathrm{JT}}=350 \mathrm{~K}$. We estimate $\Delta T_{\mathrm{JT}}$ from our model to understand how such a drastic change of the JT ordering temperature can occur by the increase in chemical pressure.

We rewrite $E_{\mathrm{tot}}^{\min }$ in Eq. (27) for $\tilde{p}>\tilde{p}_{c}$ as follows.

$$
E_{\mathrm{tot}}^{\min }=-\frac{\tilde{p}^{2}}{2\left(a_{1}+a_{2}\right)}-\frac{\lambda^{2}}{2 a_{1}}-\frac{a_{1}\left(\tilde{p}-\tilde{p}_{c}\right)^{2}}{2\left(a_{1}+a_{2}\right)^{2}},
$$

where

$$
\begin{aligned}
\tilde{p}_{c} & =\tilde{p}_{c}^{*}-\delta \tilde{p}_{c}, \\
\delta \tilde{p}_{c} & =\frac{\lambda^{2}}{4 b_{1} a_{1}}\left(a_{1}+a_{2}\right) .
\end{aligned}
$$

Since $\delta \tilde{p}_{c}$, the change in the critical chemical pressure due to the $E_{\mathrm{JT}}$ term, is small relative to $\tilde{p}-\tilde{p}_{c}^{*}$, with $\delta \tilde{p}_{c} /\left(\tilde{p}-\tilde{p}_{c}^{*}\right) \approx 0.3$ for parameter values in Sect. III, we keep the terms up to linear in $\delta \tilde{p}_{c}$ only and rewrite according to the origin of each term as follows.

$$
\begin{aligned}
E_{\mathrm{tot}}^{\min } & \approx E_{\mathrm{comp}}^{\min }+E_{\mathrm{JT}}^{\min }+E_{\mathrm{bk}}^{\min }+E_{\mathrm{bk}, \mathrm{JT}, \mathrm{sh}}^{\min }, \\
E_{\mathrm{comp}}^{\min } & =-\frac{1}{2} \frac{\tilde{p}^{2}}{a_{1}+a_{2}}, \\
E_{\mathrm{JT}}^{\min } & =-\frac{1}{2} \frac{\lambda^{2}}{a_{1}}, \\
E_{\mathrm{bk}}^{\min } & =-\frac{1}{2} \frac{a_{1}}{\left(a_{1}+a_{2}\right)^{2}}\left(\tilde{p}-\tilde{p}_{c}^{*}\right)^{2}, \\
E_{\mathrm{bk}, \mathrm{JT}, \mathrm{sh}}^{\min } & =-\frac{a_{1}}{\left(a_{1}+a_{2}\right)^{2}}\left(\tilde{p}-\tilde{p}_{c}^{*}\right) \delta \tilde{p}_{c}, \\
& =-\frac{\lambda^{2}}{4 b_{1}\left(a_{1}+a_{2}\right)}\left(\tilde{p}-\tilde{p}_{c}^{*}\right) .
\end{aligned}
$$

The first three terms, $E_{\mathrm{comp}}^{\mathrm{min}}, E_{\mathrm{JT}}^{\mathrm{min}}$, and $E_{\mathrm{bk}}^{\mathrm{min}}$, represent the energy terms purely due to compression, JT distortion, and buckling, respectively. The fourth term is the energy due to the coherent buckling, JT and shear distortions, indicated by its dependence on $\tilde{p}-\tilde{p}_{c}^{*}$, $\lambda$ and $b_{1}$, which gives extra stability to the JT ordering due to the chemical pressure.

To estimate $T_{\mathrm{JT}}$, we consider a high temperature state with random JT distortions, for which the energy can be written in a similar way as Eq. (32) except for the absence of the fourth term due to the lack of coherence among distortions as explained in Sect. VA,

$$
E_{\mathrm{tot}}^{\mathrm{ran}}=E_{\mathrm{comp}}^{\mathrm{ran}}+E_{\mathrm{JT}}^{\mathrm{ran}}+E_{\mathrm{bk}}^{\mathrm{ran}}
$$


We expect $E_{\mathrm{comp}}^{\mathrm{ran}} \approx E_{\mathrm{comp}}^{\mathrm{min}}$ and $E_{\mathrm{bk}}^{\mathrm{ran}} \approx E_{\mathrm{bk}}^{\mathrm{min}}$ since the unit cell volume and buckling angle do not change very much as the temperature crosses $T_{\mathrm{JT}}$ (Ref. 21). Therefore, the energy difference between JT ordered and JT disordered state is

$$
E_{\mathrm{tot}}^{\mathrm{ran}}-E_{\mathrm{tot}}^{\mathrm{min}} \approx E_{\mathrm{JT}}^{\mathrm{ran}}-E_{\mathrm{JT}}^{\mathrm{min}}-E_{\mathrm{bk}, \mathrm{JT}, \mathrm{sh}}^{\mathrm{min}}
$$

We first verify that our model gives the correct order of magnitude of $T_{\mathrm{JT}}$ itself. An order of magnitude estimate for $T_{\mathrm{JT}}$ can be made from the energy difference between two different JT ordered states, one the most favored state and the other relatively unfavored state. The most favored state is that with the JT distortion of $\vec{k}=(\pi, \pi)$ considered so far in this paper and has the JT energy of $E_{\mathrm{JT}}^{\min }=-\lambda^{2} /\left(2 a_{1}\right)$. We choose a state with the same size of JT distortion $e_{3}$ but with a wave vector $\vec{k}=(0,0)$ as a relatively unfavored state, with energy $E_{\mathrm{JT}}^{\text {unif }}=-\lambda^{2} /\left[2\left(a_{1}+a_{2}\right)\right]$. Using the estimated parameter values, $a_{1}=150 \mathrm{eV}$, $a_{2}=30-80 \mathrm{eV}, \lambda=10 \mathrm{eV}$, we obtain $E_{\mathrm{JT}}^{\text {unif }}-E_{\mathrm{JT}}^{\min } \approx 600-1300 \mathrm{~K}$, which has the same order of magnitude as the experimentally observed $T_{\mathrm{JT}}$ in the range of $750 \mathrm{~K}-1100 \mathrm{~K}$.

For the change in $T_{\text {JT }}$ between $\mathrm{LaMnO}_{3}$ and $\mathrm{NdMnO}_{3}$, the only term in Eq. (38)

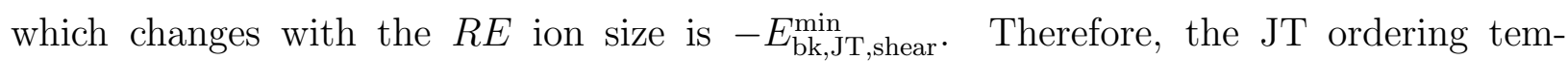
perature variation between $\mathrm{LaMnO}_{3}$ and $\mathrm{NdMnO}_{3}$ can be related to $-E_{\mathrm{bk}, \mathrm{JT}, \mathrm{sh}}^{\mathrm{min}}\left(\mathrm{NdMnO}_{3}\right)+$ $E_{\mathrm{bk}, \mathrm{JT}, \mathrm{sh}}^{\min }\left(\mathrm{LaMnO}_{3}\right)$ within a factor of the order of one. We express $E_{\mathrm{bk}, \mathrm{JT}, \mathrm{sh}}^{\mathrm{min}}$ in terms of $\left(e_{3 s}^{\prime}\right)^{\min }$,

$$
E_{\mathrm{bk}, \mathrm{JT}, \mathrm{sh}}^{\min }=-\frac{1}{2} \frac{\lambda^{2}}{4 b_{1}}\left[\left(e_{3 s}^{\prime}\right)^{\min }\right]^{2}
$$

According to the experimental data, ${ }^{21,22,27}$ the Mn-O-Mn bond angle is $155^{\circ}$ for $\mathrm{LaMnO}_{3}$ and $150^{\circ}$ for $\mathrm{NdMnO}_{3}$, which corresponds to $\left(e_{3 s}^{\prime}\right)^{\min }$ of about 0.22 and 0.27 , respectively. These distortions, along with parameter values $\lambda=10 \mathrm{eV}$ and $b_{1}=20-25 \mathrm{eV}$, result in $-E_{\mathrm{bk}, \mathrm{JT}, \mathrm{sh}}^{\min }\left(\mathrm{NdMnO}_{3}\right)+E_{\mathrm{bk}, \mathrm{JT}, \mathrm{sh}}^{\min }\left(\mathrm{LaMnO}_{3}\right) \approx 12-16 \mathrm{meV} \approx 140-190 \mathrm{~K}$. From a classical Monte Carlo simulation for the double-well potential model in Ref. 15, we find that the structural ordering temperature is about twice the energy difference between the distorted ground state and undistorted high energy state. ${ }^{28}$ Although such a relation would depend on the details of the model, if we assume a similar situation in the current model, the JT ordering temperature variation can be estimated as twice the energy difference, therefore, $T_{\mathrm{JT}}\left(\mathrm{NdMnO}_{3}\right)-T_{\mathrm{JT}}\left(\mathrm{LaMnO}_{3}\right) \approx 2 \times\left[-E_{\mathrm{bk}, \mathrm{JT}, \mathrm{sh}}^{\min }\left(\mathrm{NdMnO}_{3}\right)+E_{\mathrm{bk}, \mathrm{JT}, \mathrm{sh}}^{\min }\left(\mathrm{LaMnO}_{3}\right)\right]=280-$ $380 \mathrm{~K}$, which agrees well with the experimental change in $T_{\mathrm{JT}}, 350 \mathrm{~K}$. 
This agreement shows that indeed the JT ordered state is more stabilized when the buckling increases for smaller rare earth ions for undoped compounds. The relatively large increase in the JT ordering temperature, both in theory and experimental data, shows that the interplay between the rare earth ion size and the JT distortion is significant, and should be taken into account to explain the well-known temperature-tolerance factor phase diagram of both undoped and doped perovskite manganites.

\section{Relation between shear, buckling, and deviatoric distortion}

Equations (22) and (25) imply that the following quantities remain constant regardless of the variation in chemical pressure:

$$
\begin{aligned}
\left(e_{3 s}\right)^{\min } & =\frac{\lambda}{a_{1}}, \\
\frac{\left(e_{20}\right)^{\min }}{\left(e_{3 s}^{\prime}\right)^{\min }} & =\frac{\lambda}{4 b_{1}}, \\
\frac{\left(e_{20}\right)^{\min }}{\left(e_{3 s}\right)^{\min }\left(e_{3 s}^{\prime}\right)^{\min }} & =\frac{a_{1}}{4 b_{1}} .
\end{aligned}
$$

We calculate these quantities from the experimental data for $\mathrm{LaMnO}_{3}$ and $\mathrm{NdMnO}_{3}$, and

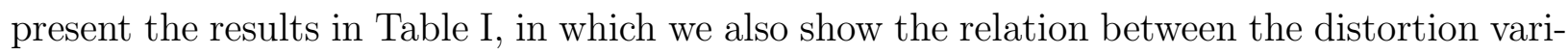
ables in our model and experimental parameters and the estimate of $T_{\mathrm{JT}}$ and $\Delta T_{\mathrm{JT}}$ obtained in Sect. VB. The results show that $\left(e_{3 s}\right)^{\min },\left(e_{20}\right)^{\min } /\left(e_{3 s}^{\prime}\right)^{\min }$, and $\left(e_{20}\right)^{\min } /\left[\left(e_{3 s}\right)^{\min }\left(e_{3 s}^{\prime}\right)^{\min }\right]$ remain constant within $2 \%, 10 \%$, and $7 \%$, respectively, in spite of $32 \%$ and $19 \%$ changes in $\left(e_{20}\right)^{\mathrm{min}}$ and $\left(e_{3 s}^{\prime}\right)^{\mathrm{min}}$. These values also agree well with theoretical estimates obtained from the parameters in Sect!II, The results underscore the strong coupling between these distortions, in particular, the important role played by the uniform shear distortion in connecting the Jahn-Teller and buckling distortions, an aspect neglected in the literature so far.

\section{CONCLUSION}

From the analysis of a Keating energy expression expanded in terms of the atomic-scale symmetry-modes, we find that the effect of small rare earth ion size, known as chemical pressure effect, is significant in stabilizing the long range Jahn-Teller distortion in undoped 
TABLE I. Parameters from experimental data and comparison with theoretical estimates. Experimental data for the lattice constants, bond lengths, and bond angles for $\mathrm{LaMnO}_{3}$ and $\mathrm{NdMnO}_{3}$ are from Ref. 21 and 22 , respectively, measured at room temperature.

\begin{tabular}{l|ll|c}
\hline \hline Parameters & $\mathrm{LaMnO}_{3} \mathrm{NdMnO}_{3}$ & Theoretical estimates \\
\hline Lattice constant, $a$ & $5.54 \AA$ & $5.414 \AA$ & \\
Lattice constant, $b$ & $5.75 \AA$ & $5.731 \AA$ & \\
Long Mn-O bond length within $a b$ plane, $l$ & $2.718 \AA$ & $2.20 \AA$ & \\
Short Mn-O bond length within $a b$ plane, $s$ & $1.907 \AA$ & $1.90 \AA$ & \\
Mn-O-Mn bond angle within $a b$ plane, $\varphi$ & $155.1^{\circ}$ & $149.8^{\circ}$ & \\
\hline $\mathrm{Mn}-\mathrm{Mn}$ distance with $e_{10}$ only, $(a+b) /(2 \sqrt{2})$ & $3.995 \AA$ & $3.94 \AA$ & Compressed from $u \approx 4 \AA$ \\
\hline$e_{20}=(b-a) /(2 \sqrt{2} u)$ & 0.019 & 0.028 & \\
$e_{3 s}^{\prime}=(\pi-\varphi) / 2$ & 0.22 & 0.27 & \\
$e_{20} / e_{3 s}^{\prime}$ & 0.09 & 0.10 & $\lambda /\left(4 b_{1}\right)=0.10-0.13$ \\
$e_{\mathrm{JT}}=(l-s) / u$ & 0.068 & 0.075 & \\
$e_{3 s} \approx e_{\mathrm{JT}}-e_{20} e_{3 s}^{\prime}$ & 0.064 & 0.067 & $\lambda / a_{1}=0.067$ \\
$e_{20} /\left(e_{3 s} e_{3 s}^{\prime}\right)$ & 1.4 & 1.5 & $a_{1} /\left(4 b_{1}\right)=1.5-1.9$ \\
\hline$T_{\mathrm{JT}}$ & $750 \mathrm{~K}$ & $1100 \mathrm{~K}$ & $E_{\mathrm{JT}}^{\text {unif }}-E_{\mathrm{JT}}^{\mathrm{min}}=600-1300 \mathrm{~K}$ \\
$\Delta T_{\mathrm{JT}}$ & \multicolumn{2}{|l}{$350 \mathrm{~K}$} & $-2 \Delta E_{\mathrm{bk}, \mathrm{JT}, \mathrm{sh}}^{\min }=280-380 \mathrm{~K}$ \\
\hline \hline
\end{tabular}

perovskite manganites. We obtain good agreement with the experimental data on the JahnTeller ordering temperature and the substantial increase of the Jahn-Teller ordering temperature from $\mathrm{LaMnO}_{3}$ to $\mathrm{NdMnO}_{3}$. We propose that similar effects need to be considered to understand the phase diagram for doped perovskite manganites. We also explain the appearance of the uniform shear distortion below the Jahn-Teller ordering temperature in terms of the coupling between coherent shear, buckling, and deviatoric distortions within the Jahn-Teller energy. Moreover, we estimate the ratio between these distortions at low temperature, and find good agreement with experimental data for $\mathrm{LaMnO}_{3}$ and $\mathrm{NdMnO}_{3}$, which confirms the coupling proposed between them in our model.

This work was supported by US DOE/LANL Award No. DE-AC52-06NA25396/1705901 (T.F.S., K.H.A.), ANL XSD Visitor Program (K.H.A.), NJIT (T.F.S., K.H.A.), US DOE 
LANL LDRD (T.L., A.S., A.R.B.), and DOE FWP 70069 (P.B.L.).

1 R. von Helmolt, J. Wecker, B. Holzapfel, L. Schultz, and K. Samwer Phys. Rev. Lett. 71, 2331 (1993).

2 S. Jin, T. H. Tiefel, M. McCormack, R. A. Fastnacht, R. Ramesh, and L. H. Chen, Science 264, $413(1994)$.

3 A. J. Millis, P. B. Littlewood, and B. I. Shraiman, Phys. Rev. Lett. 74, 5144 (1995).

4 A. J. Millis, Phys. Rev. B 53, 8434 (1996).

5 H. Röder, J. Zang, and A. R. Bishop, Phys. Rev. Lett. 76, 1356 (1996).

6 M. B. Salamon and M. Jaime, Rev. Mod. Phys. 73, 583 (2001).

7 H. Y. Hwang, S.-W. Cheong, P. G. Radaelli, M. Marezio, and B. Batlogg, Phys. Rev. Lett. 75, 914 (1995).

8 M. O. Dzero, L. P. Gorkov, and V. Z. Kresin, Eur. Phys. J. B 14, 459 (2000).

9 J. A. Fernandez-Baca, P. Dai, H. Y. Hwang, C. Kloc, and S.-W. Cheong, Phys. Rev. Lett. 80, $4012(1998)$.

10 J. W. Liu, Z. Zeng, Q. Q. Zheng, and H. Q. Lin, Phys. Rev. B 60, 12968 (1999).

11 J. W. Lynn, R. W. Erwin, J. A. Borchers, Q. Huang, A. Santoro, J.-L. Peng, and Z. Y. Li, Phys. Rev. Lett. 76, 4046 (1996).

12 P. G. Radaelli, G. Iannone, M. Marezio, H. Y. Hwang, S.-W. Cheong, J. D. Jorgensen, and D. N. Argyriou, Phys. Rev. B 56, 8265 (1997).

13 D. Louca, T. Egami, W. Dmowski, and J. F. Mitchell, Phys. Rev. B 64, 180403(R) (2001).

14 T. Kimura, T. Goto, H. Shintani, K. Ishizaka, T. Arima, and Y. Tokura, Nature 426, 55 (2003).

15 K. H. Ahn, T. Lookman, A. Saxena, and A. R. Bishop, Phys. Rev. B 68, 092101 (2003).

16 K. H. Ahn, T. Lookman, and A. R. Bishop, Nature (London) 428, 401 (2004).

17 The normalization factor is chosen in such a way that, for example, $e_{3}=2 d_{\mathrm{O}}$ if each $\mathrm{O}$ ion around $\mathrm{Mn}$ ion is displaced by $d_{\mathrm{O}}$ in the way shown in Fig. 3 ,

18 P. N. Keating, Phys. Rev. 145, 637 (1966).

19 P. B. Littlewood, Phys. Rev. B 34, 1363 (1986).

20 K. H. Ahn and A. J. Millis, Phys. Rev. B 64, 115103 (2001).

21 J. Rodríguez-Carvajal, M. Hennion, F. Moussa, A. H. Moudden, L. Pinsard, and A. 
Revcolevschi, Phys. Rev. B 57, R3189 (1998).

22 A. M. Balagurov, S. N. Bushmeleva, V. Yu. Pomjakushin, D. V. Sheptyakov, V. A. Amelichev, O. Yu. Gorbenko, A. R. Kaul, E. A. Gan'shina, and N. B. Perkins, Phys. Rev. B 70, 014427 (2004).

23 T. W. Darling, A. Migliori, E. G. Moshopoulou, S. A. Trugman, J. J. Neumeier, J. L. Sarrao, A. R. Bishop, and J. D. Thompson, Phys. Rev. B 57, 5093 (1998).

24 N. W. Ashcroft and N. D. Mermin, Solid State Physics (Holt, Rinehart and Winston, New York, 1976), pp. 443-447.

25 A. P. Mirgorodsky and M. B. Smirnov, J. Phys: Condens. Matter 5, 3313 (1993).

26 J. Zhang, P. Dai, J. A. Fernandez-Baca, E. W. Plummer, Y. Tomioka, and Y. Tokura, Phys. Rev. Lett. 86, 3823 (2001).

27 T. Kimura, S. Ishihara, H. Shintani, T. Arima, K. T. Takahashi, K. Ishizaka, and Y. Tokura, Phys. Rev. B 68, 060403(R) (2003).

28 We carry out classical Monte Carlo simulations with the same energy expression and parameter values used for the results in Fig. 3 in Ref. 15. We find that the structural phase transition temperature is about 0.15 , which is about twice the depth of the potential well 0.08 . 